\title{
FOOD ADVERTISING TARGETTING YOUNG CONSUMERS- WHAT DO PARENTS THINK
}

\author{
DR. SUNAYNA KHURANA \& KANUPRIYA DANG \\ Professor, CBSA, Chandigarh Group of Colleges, Landran, Punjab, India \\ Research Scholar, I.K. Gujral Punjab Technical University, Kapurthala, Punjab, India
}

\begin{abstract}
Purpose - This study aims to highlight the parents' viewpoints regarding the measures which can be adopted to curb the harmful impact concerning food advertising.

Design/Methodology/Approach - A descriptive research design of children (aged 5-12 years) and a survey of the parents of the concerned children was undertaken.

Findings - Parents believed that by encouraging children towards sports/ physical activities and through various regulatory measures the negative impact of advertising could be restricted.

Research Limitations/Implications - This research shows that various steps could be adopted to restrict the negative impact of food advertising on children that can be considered to undertake future research to provide more in-depth understanding of the impact of advertising on children's preferences for food and health.

Originality/value - The findings highlight the measures required to be adopted to decrease the negative impact concerning food advertising upon children

KEYWORDS: Advertising, Food, Marketing, Parent, Role
\end{abstract}

Received: Jun 08, 2020; Accepted: Jun 28, 2020; Published: Sep 15, 2020; Paper Id.: IJMPERDJUN20201240

\section{INTRODUCTION}

All marketing activities now days revolve around children. Numerous tactics are used by marketers now a days to crate a significant and beneficial segment of the market. Television continues to persist as the prominent medium for marketing with regard to children. Advertisements on television are regarded as instructive as children collect information related to different products and it is convenient for them to collect information and assess the brands as well. Nevertheless, the aptitude for comprehending the messages conveyed in advertisements and the intent of such ads widens with age.(Boush et al. 1994; John, 1999; Soni, P. \& Singh, R. (2012). Children these days are beleaguered by advertisements as they are exposed to them to a great extent (Rozendaal et al., 2011). Food these days corresponds to the second most advertised product (Kelly et al., 2010). Food is one such product that has experienced a reflective change since past few years to allure kids through various strategies (De la Ville et al.,2010). Food advertising is by and large inclined towards unhealthy options( Hingle et al., 2015) and due to the relative significance of children for both the marketers as well as the policy makers( Cook, 2009) the aptitude of children to comprehend advertisements is considered central to scholars' research regarding effects of marketing communication on the children(Mc Alister and Bargh, 2016). Young children are predominantly influenced by ads. Further they make requests to their parents for a range of products and keep on demanding and pestering till their 
parents get them those products. Parents on the other hand are not always keen/able to fulfill their demands. It has been found in various researches that a larger percentage of advertisements are aired during the kids viewing times .(George 2003; Kaiser Family Foundation 2004) and a majority of them are for unhealthy food products. (Kotz and Story, 1994; Byrd-Bredbenner and Grasso, 1999; Consumers International, 2004, 2008; Hardy et al., 2006; Maher et al., 2006; Jenkin et al., 2008; Stitt and Kunkel, 2008;Soni, P., \& Singh, R. 2012).

There are numerous advertisements that are broadcast during the times when children watch their programs. These advertisements to a large extent have an impact on their food preferences and eating habits. As children watch a large number of ads on different channels daily in India (George, 2003), they are open to quite a lot of commercials for food that increases the propensity of consuming advertised foods (Miryala, 2011). Consumption of unhealthy food has an adverse effect on a child and also leads to health related problems. Excessive intake of such less healthy foods tends to raise the incidence of health relatedproblems in children namelyobesity, diabetes, heart attack and even fatal diseases like cancer. Therefore, some measures must to be taken to protect children from food advertising. Parents therefore are active players in mediating TV viewing by their children. The parents of such children are greatly apprehensive about their child's future. The present study highlights parents' viewpoints regarding what all measures can be adopted to curb the menace.

\section{REVIEW OF LITERATURE}

Since ages it has been believed that parents always want toinculcate good habits in their children (Gillespie, 1989), they exercise a firm control upon their kid's diet but all in vien (Bijmolt et al., 1998) and impracticable because whenchildren are extremely exposed to advertisements on television they tend to develop a strong fondness for the foods that are less healthy (Agrawal and Tripathi, 2008; Mittal et al., 2010). Time and again if parents keep on refusing their children it may also lead to parent-child conflict. As a result, it has been found that a strict approach has to be devised that helps to lesserthe exposure that is related to food ads.

Kaur (2011) conducted a study to find out the impact of television advertising on children. A sample of 400 children and mothers was taken and data was analysed using ANOVA, factor analysis, chi square and correlation .It was identified that children who watch TV in the presence of parents have better understanding of advertisements. They have a positive yet pragmatic attitude towards TV advertisements. But the mothers held television advertisements responsible for rising materialism. The most important reason for likeability of a particular advertisement was the product/ brand.

Haroon et al. (2011) conducted a study in Pakistan and reviewed children's weekend programmes over a three week period. The study found that $35.11 \%$ of food advertisements are for snacks, and there were no advertisements for fruits.

Adya et al.(2014)explored the role of family communication and brand awareness in understanding the influence of child on purchase decisions. The sample comprised of 229 pairs of mother and child. The study was conducted in Pune and data was analyzed using SPSS. It was concluded that family demographics and family communication affect the influence of child wherein child is not a learner but in a teacher's role. Brand awareness is an important factor affecting the influence of the child.

Ussaima (2015) analysed the influence of television advertisements and the role of television advertising in creating product awareness among children. A sample of 354 children and 250 parents of Madurai city was taken using 
random sampling. Data was analysed using SPSS. It was concluded that Television advertisements are the most important source of product awareness followed by school friends and parents and 14\% children make purchase requests immediately after watching advertisements. $45.48 \%$ children made purchase requests to their father, $44.35 \%$ to mother, $7.6 \%$ to grandparents and $2.5 \%$ to siblings.

Therefore, the present research seeksto identify the various remedial measures required to apprehend the problem of adverse influence of food advertising directed at Indian children and tospecifically answer research questions, namely:

RQ1. Can the adverse influence of food advertisements be controlled through active role of Parents ?

RQ2. Are there any measures that can be adopted to restrict food advertising?

\section{RESEARCH METHODS}

The data has been collected from the cities namely Chandigarh, Panchkula and Mohali during the period November 2019February 2020. The basic sampling unit for the present study were the parents as they are regarded as the key caregivers for a child. Six schools were selected i.e. two from each city and the children were distributed the questionnaires and were asked to get them filled by their parents. Out of 150 questionnaires that were distributed, 100 complete questionnaires were considered for the study. Hence a total sample of 100 parents of children of the age group 5 to 12 years was finalised.

The inter item reliability (Cronbach's alpha) for the measure is 0.752 . SPSS 25.0 version has been utilized for data analysis. Means, standard deviations, correlations have been applied to analyse the data.

Table 1: Reliability Analysis

\begin{tabular}{|c|c|}
\hline Cronbach's Alpha & N of Items \\
\hline $\mathbf{0 . 7 5 2}$ & 11 \\
\hline
\end{tabular}

As the cronbach alpha value is above 0.60 , it means that scale is reliable.

\section{RESULT ANALYSIS}

Table 2

\begin{tabular}{|c|c|c|c|c|c|}
\hline \multicolumn{6}{|c|}{ Descriptive Statistics } \\
\hline & $\mathbf{N}$ & Minimum & Maximum & Mean & $\begin{array}{c}\text { Std. } \\
\text { Deviati } \\
\text { on }\end{array}$ \\
\hline Ad Influence & 100 & 1 & 5 & 3.62 & 0.896 \\
\hline Time Limit & 100 & 1 & 5 & 3.74 & 1.203 \\
\hline Active Role Of Parents & 100 & 1 & 5 & 4.40 & 0.985 \\
\hline School Role & 100 & 4 & 5 & 4.50 & 0.503 \\
\hline Parental Interaction & 100 & 4 & 5 & 4.52 & 0.502 \\
\hline Sports/ Physical Activities & 100 & 4 & 5 & 4.78 & 0.416 \\
\hline Govt Regulations & 100 & 4 & 5 & 4.44 & 0.499 \\
\hline Restrictions On Food Ads & 100 & 3 & 5 & 4.18 & 0.770 \\
\hline Ban On Food Ads & 100 & 1 & 5 & 2.92 & 1.002 \\
\hline $\begin{array}{l}\text { Ban On Ads Promoting Unhealthy } \\
\text { Food }\end{array}$ & 100 & 2 & 5 & 4.10 & 0.990 \\
\hline $\begin{array}{l}\text { Ban On Food Ads When Kids Watch } \\
\text { Tv }\end{array}$ & 100 & 2 & 5 & 4.14 & 0.779 \\
\hline Valid N (Listwise) & 100 & & & & \\
\hline
\end{tabular}


From the above table it can be noted the mean of Sports/ Physical Activities is the highest (4.78) followed by parental interaction (4.52) and school's role (4.50) is important to restrict the adverse effect of advertising. The mean of government regulation (4.44) and restrictions on food advertisements (4.18) are noted. Hence it depicts the viewpoint of the parents. Parents believe that by encouraging children into sports and other physical activities and by interacting with them frequently on the adverse effects of advertising the problem can be handled in a positive regard. Further it has been opined that the role of the school/teachers in inculcating good/healthy eating habits among children is quite relevant as in the earlier years the children tend to emulate and follow whatever is told by their school/teachers. Moreover parents believe that government should enforce strict restrictions concerning food advertising to children. They also believe that a ban on food ads which promote unhealthy foods should be introduced during the viewing hours of children.

Table 3: Correlation

\begin{tabular}{|c|c|c|c|c|c|c|c|c|c|c|c|c|}
\hline Variables & \multicolumn{2}{|c|}{$\begin{array}{c}\text { Ad } \\
\text { Influence }\end{array}$} & $\begin{array}{c}\mathrm{Ti} \\
\mathbf{m e} \\
\mathbf{L i} \\
\mathbf{m i} \\
\mathbf{t}\end{array}$ & $\begin{array}{c}\text { Acti } \\
\text { ve } \\
\text { Rol } \\
\text { e Of } \\
\text { Par } \\
\text { ents }\end{array}$ & $\begin{array}{c}\text { Role Of } \\
\text { School/T } \\
\text { eachers }\end{array}$ & $\begin{array}{c}\text { Paren } \\
\text { tal } \\
\text { Intera } \\
\text { ction }\end{array}$ & $\begin{array}{c}\text { Sports/P } \\
\text { hysical } \\
\text { Activitie } \\
\text { s }\end{array}$ & $\begin{array}{l}\text { Govt } \\
\text { Regul } \\
\text { ations }\end{array}$ & $\begin{array}{l}\text { Restri } \\
\text { ctions } \\
\text { On } \\
\text { Food } \\
\text { Ads }\end{array}$ & $\begin{array}{c}\text { Ba } \\
\text { n } \\
\text { On } \\
\text { Foo } \\
\text { d } \\
\text { Ad } \\
\text { s }\end{array}$ & $\begin{array}{c}\text { Ban } \\
\text { On } \\
\text { Ads } \\
\text { Prom } \\
\text { oting } \\
\text { Unhe } \\
\text { althy } \\
\text { Food }\end{array}$ & $\begin{array}{c}\text { Ba } \\
\text { n } \\
\text { On } \\
\text { Foo } \\
\text { d } \\
\text { Ads } \\
\text { Wh } \\
\text { en } \\
\text { Kid } \\
\text { s } \\
\text { Wa } \\
\text { tch } \\
\text { Tv }\end{array}$ \\
\hline \multirow[t]{2}{*}{$\begin{array}{l}\text { Ad } \\
\text { Influence }\end{array}$} & $\begin{array}{l}\text { Pearso } \\
\mathrm{n} \\
\text { Correla } \\
\text { tion } \\
\end{array}$ & 1 & $\begin{array}{l}.39 \\
5^{* *}\end{array}$ & .494 & -0.022 & -0.005 & 0.099 & $.332^{* *}$ & 0.188 & $\begin{array}{c}0.1 \\
68\end{array}$ & $.408^{* *}$ & .28 \\
\hline & $\begin{array}{l}\text { Sig. (2- } \\
\text { tailed) }\end{array}$ & & $\begin{array}{c}0.0 \\
00 \\
\end{array}$ & $\begin{array}{c}0.00 \\
0 \\
\end{array}$ & 0.825 & 0.958 & 0.329 & 0.001 & 0.061 & $\begin{array}{l}0.0 \\
94 \\
\end{array}$ & 0.000 & $\begin{array}{c}0.0 \\
05 \\
\end{array}$ \\
\hline \multirow[t]{2}{*}{$\begin{array}{l}\text { Time } \\
\text { Limit }\end{array}$} & $\begin{array}{l}\text { Pearso } \\
\mathrm{n} \\
\text { Correla } \\
\text { tion }\end{array}$ & $\begin{array}{l}.39 \\
5^{* *}\end{array}$ & 1 & .686 & -0.184 & 0.193 & $.288^{* * *}$ & 0.125 & $.465^{* *}$ & $\begin{array}{l}0.0 \\
83\end{array}$ & $.582^{* *}$ & .27 \\
\hline & $\begin{array}{l}\text { Sig. (2- } \\
\text { tailed) }\end{array}$ & $\begin{array}{l}0.0 \\
00 \\
\end{array}$ & & $\begin{array}{c}0.00 \\
0\end{array}$ & 0.067 & 0.055 & 0.004 & 0.214 & 0.000 & $\begin{array}{c}0.4 \\
11 \\
\end{array}$ & 0.000 & $\begin{array}{c}0.0 \\
05 \\
\end{array}$ \\
\hline \multirow[t]{2}{*}{$\begin{array}{l}\text { Active } \\
\text { role of } \\
\text { Parents }\end{array}$} & $\begin{array}{l}\text { Pearso } \\
\mathrm{n} \\
\text { Correla } \\
\text { tion } \\
\end{array}$ & $\begin{array}{l}.49 \\
4^{* *}\end{array}$ & $\begin{array}{l}.68 \\
6^{* *}\end{array}$ & 1 & -0.082 & -0.180 & 0.118 & $.214^{*}$ & $.224^{*}$ & $\begin{array}{l}.38 \\
1^{* *}\end{array}$ & $.518^{* *}$ & $\begin{array}{l}0.0 \\
32\end{array}$ \\
\hline & $\begin{array}{l}\text { Sig. (2- } \\
\text { tailed) }\end{array}$ & $\begin{array}{l}0.0 \\
00\end{array}$ & $\begin{array}{l}0.0 \\
00\end{array}$ & & 0.419 & 0.073 & 0.241 & 0.033 & 0.025 & $\begin{array}{c}0.0 \\
00\end{array}$ & 0.000 & $\begin{array}{l}0.7 \\
55 \\
\end{array}$ \\
\hline \multirow{2}{*}{$\begin{array}{l}\text { Role of } \\
\text { school/T } \\
\text { eachers }\end{array}$} & $\begin{array}{l}\text { Pearso } \\
\mathrm{n} \\
\text { Correla } \\
\text { tion }\end{array}$ & $\begin{array}{c}- \\
0.0 \\
22\end{array}$ & $\begin{array}{l}0.1 \\
84\end{array}$ & $\begin{array}{c}- \\
0.08 \\
2\end{array}$ & 1 & $.400^{* *}$ & 0.145 & $.242^{*}$ & -0.183 & $\begin{array}{l}- \\
.28 \\
1^{* *}\end{array}$ & -0.061 & $\begin{array}{c}- \\
0.0 \\
77\end{array}$ \\
\hline & $\begin{array}{l}\text { Sig. (2- } \\
\text { tailed) }\end{array}$ & $\begin{array}{l}0.8 \\
25\end{array}$ & $\begin{array}{l}0.0 \\
67\end{array}$ & $\begin{array}{c}0.41 \\
9\end{array}$ & & 0.000 & 0.150 & 0.015 & 0.069 & $\begin{array}{c}0.0 \\
05\end{array}$ & 0.547 & $\begin{array}{c}0.4 \\
44\end{array}$ \\
\hline $\begin{array}{l}\text { Parental } \\
\text { Interactio } \\
\mathrm{n}\end{array}$ & $\begin{array}{l}\text { Pearso } \\
\mathrm{n} \\
\text { Correla } \\
\text { tion }\end{array}$ & $\begin{array}{c}- \\
0.0 \\
05\end{array}$ & $\begin{array}{l}0.1 \\
93\end{array}$ & $\begin{array}{c}- \\
0.18 \\
0\end{array}$ & $.400^{* * *}$ & 1 & $.359^{* * *}$ & $.448^{* * *}$ & $.382^{* *}$ & $\begin{array}{l}- \\
.63 \\
9^{* *}\end{array}$ & $.260^{* *}$ & $\begin{array}{l}.22 \\
5^{*}\end{array}$ \\
\hline
\end{tabular}




\begin{tabular}{|c|c|c|c|c|c|c|c|c|c|c|c|c|}
\hline & $\begin{array}{l}\text { Sig. (2- } \\
\text { tailed) }\end{array}$ & $\begin{array}{c}0.9 \\
58\end{array}$ & $\begin{array}{c}0.0 \\
55\end{array}$ & $\begin{array}{c}0.07 \\
3\end{array}$ & 0.000 & & 0.000 & 0.000 & 0.000 & $\begin{array}{c}0.0 \\
00\end{array}$ & 0.009 & $\begin{array}{l}0.0 \\
24\end{array}$ \\
\hline \multirow{2}{*}{$\begin{array}{l}\text { Sports/ph } \\
\text { ysical } \\
\text { activities }\end{array}$} & $\begin{array}{l}\text { Pearso } \\
\mathrm{n} \\
\text { Correla } \\
\text { tion }\end{array}$ & $\begin{array}{l}0.0 \\
99\end{array}$ & $\begin{array}{l}.28 \\
8^{* * *}\end{array}$ & $\begin{array}{c}0.11 \\
8\end{array}$ & 0.145 & $.359^{* *}$ & 1 & 0.082 & $.251^{*}$ & $\begin{array}{l}0.1 \\
39\end{array}$ & 0.152 & $\begin{array}{l}.34 \\
5^{* * *}\end{array}$ \\
\hline & $\begin{array}{l}\text { Sig. (2- } \\
\text { tailed) }\end{array}$ & $\begin{array}{l}0.3 \\
29 \\
\end{array}$ & $\begin{array}{c}0.0 \\
04 \\
\end{array}$ & $\begin{array}{c}0.24 \\
1\end{array}$ & 0.150 & 0.000 & & 0.419 & 0.012 & $\begin{array}{l}0.1 \\
66\end{array}$ & 0.131 & $\begin{array}{l}0.0 \\
00\end{array}$ \\
\hline \multirow{2}{*}{$\begin{array}{l}\text { Govt } \\
\text { regulatio } \\
\text { ns }\end{array}$} & $\begin{array}{l}\text { Pearso } \\
\mathrm{n} \\
\text { Correla } \\
\text { tion }\end{array}$ & $\begin{array}{l}.33 \\
2^{* *}\end{array}$ & $\begin{array}{l}0.1 \\
25\end{array}$ & .214 & $.242^{*}$ & $.448^{* *}$ & 0.082 & 1 & $.317^{* * *}$ & $\begin{array}{l}0.0 \\
91\end{array}$ & $.360^{* *}$ & $\begin{array}{l}0.0 \\
48\end{array}$ \\
\hline & $\begin{array}{l}\text { Sig. (2- } \\
\text { tailed) }\end{array}$ & $\begin{array}{c}0.0 \\
01 \\
\end{array}$ & $\begin{array}{l}0.2 \\
14 \\
\end{array}$ & $\begin{array}{c}0.03 \\
3 \\
\end{array}$ & 0.015 & 0.000 & 0.419 & & 0.001 & $\begin{array}{l}0.3 \\
70 \\
\end{array}$ & 0.000 & $\begin{array}{l}0.6 \\
36 \\
\end{array}$ \\
\hline \multirow{2}{*}{$\begin{array}{l}\text { Restricti } \\
\text { ons on } \\
\text { Food } \\
\text { Ads }\end{array}$} & $\begin{array}{l}\text { Pearso } \\
\mathrm{n} \\
\text { Correla } \\
\text { tion } \\
\end{array}$ & $\begin{array}{c}0.1 \\
88\end{array}$ & $\begin{array}{l}.46 \\
5^{* *}\end{array}$ & .224 & -0.183 & $.382^{* *}$ & $.251^{*}$ & $.317^{* *}$ & 1 & $\begin{array}{l}.22 \\
8^{*}\end{array}$ & $.691^{* *}$ & $\begin{array}{l}.59 \\
7^{* * *}\end{array}$ \\
\hline & $\begin{array}{l}\text { Sig. (2- } \\
\text { tailed) }\end{array}$ & $\begin{array}{c}0.0 \\
61 \\
\end{array}$ & $\begin{array}{c}0.0 \\
00\end{array}$ & $\begin{array}{c}0.02 \\
5\end{array}$ & 0.069 & 0.000 & 0.012 & 0.001 & & $\begin{array}{l}0.0 \\
22\end{array}$ & 0.000 & $\begin{array}{l}0.0 \\
00\end{array}$ \\
\hline \multirow[t]{2}{*}{$\begin{array}{l}\text { Ban on } \\
\text { food Ads }\end{array}$} & $\begin{array}{l}\text { Pearso } \\
\mathrm{n} \\
\text { Correla } \\
\text { tion }\end{array}$ & $\begin{array}{c}0.1 \\
68\end{array}$ & $\begin{array}{l}0.0 \\
83\end{array}$ & .381 & $-.281^{* *}$ & $-.639^{* *}$ & -0.139 & -0.091 & $.228^{*}$ & 1 & 0.130 & $\begin{array}{l}0.0 \\
40\end{array}$ \\
\hline & $\begin{array}{l}\text { Sig. (2- } \\
\text { tailed) }\end{array}$ & $\begin{array}{c}0.0 \\
94 \\
\end{array}$ & $\begin{array}{c}0.4 \\
11\end{array}$ & $\begin{array}{c}0.00 \\
0\end{array}$ & 0.005 & 0.000 & 0.166 & 0.370 & 0.022 & & 0.196 & $\begin{array}{l}0.6 \\
90 \\
\end{array}$ \\
\hline \multirow{2}{*}{$\begin{array}{l}\text { Ban on } \\
\text { Ads } \\
\text { promotin } \\
\mathrm{g} \\
\text { unhealth } \\
\mathrm{y} \text { food }\end{array}$} & $\begin{array}{l}\text { Pearso } \\
\mathrm{n} \\
\text { Correla } \\
\text { tion } \\
\end{array}$ & $\begin{array}{l}.40 \\
8^{* *}\end{array}$ & .58 & .518 & -0.061 & $.260^{* * *}$ & 0.152 & $.360^{* * *}$ & $.691^{* * *}$ & $\begin{array}{l}0.1 \\
30\end{array}$ & 1 & $\begin{array}{l}.74 \\
2^{* *}\end{array}$ \\
\hline & $\begin{array}{l}\text { Sig. (2- } \\
\text { tailed) }\end{array}$ & $\begin{array}{l}0.0 \\
00\end{array}$ & $\begin{array}{l}0.0 \\
00\end{array}$ & $\begin{array}{c}0.00 \\
0\end{array}$ & 0.547 & 0.009 & 0.131 & 0.000 & 0.000 & $\begin{array}{l}0.1 \\
96 \\
\end{array}$ & & $\begin{array}{l}0.0 \\
00\end{array}$ \\
\hline \multirow{3}{*}{$\begin{array}{l}\text { Ban on } \\
\text { Food } \\
\text { Ads } \\
\text { when } \\
\text { kids } \\
\text { watch } \\
\text { TV }\end{array}$} & $\begin{array}{l}\text { Pearso } \\
\mathrm{n} \\
\text { Correla } \\
\text { tion } \\
\end{array}$ & $\begin{array}{l}.28 \\
0^{* * *}\end{array}$ & $\begin{array}{l}.27 \\
6 * *\end{array}$ & $\begin{array}{c}0.03 \\
2\end{array}$ & -0.077 & $.225^{*}$ & $.345^{* *}$ & 0.048 & $.597^{* * *}$ & $\begin{array}{c}0.0 \\
40\end{array}$ & $.742^{* *}$ & 1 \\
\hline & $\begin{array}{l}\text { Sig. (2- } \\
\text { tailed) }\end{array}$ & $\begin{array}{l}0.0 \\
05 \\
\end{array}$ & $\begin{array}{l}0.0 \\
05 \\
\end{array}$ & $\begin{array}{c}0.75 \\
5 \\
\end{array}$ & 0.444 & 0.024 & 0.000 & 0.636 & 0.000 & $\begin{array}{l}0.6 \\
90 \\
\end{array}$ & 0.000 & \\
\hline & $\mathrm{N}$ & $\begin{array}{c}10 \\
0 \\
\end{array}$ & $\begin{array}{c}10 \\
0 \\
\end{array}$ & 100 & 100 & 100 & 100 & 100 & 100 & 100 & 100 & 100 \\
\hline
\end{tabular}

From the above table it is quite evident that the adverse influence of advertisements can be restricted by resorting to time limits for watching TV and further active role of parents is necessary to bring about a change. Nonetheless the government should introduce strict restrictions and should ban unhealthy food advertising.

\section{CONCLUSIONS}

The present study attempts to identify various measures that help in apprehending the problematic situation where the negative effects of advertising are on the rise. It has been concluded that through restricting time for viewing television the impact of advertising can be controlled. Furthermore parents should play an active role in monitoring their children and help them get indulged in sports and other physical activities. Nonetheless the role of government in enforcing regulations and taking strict measures in this regard can help in dealing with this menace. 


\section{LIMITATIONS AND FURTHER RESEARCH}

Although the contribution of this study is original, yet it has a number of limitations that unlock innovative options for future research. More comprehensive research could be helpful to examine not only the factors discussed in this study but also other factors. Longitudinal study exploring the growth of advertising effectiveness, comparative analysis of urban and rural children and the viewpoints of their parents could facilitate academics and researchers better comprehend the influence of advertising with time.

\section{REFERENCES}

1. Agrawal, N. and Tripathi, A.P. (2008), “Analyzing the impact of television advertising on children's food preferences: a study of Indian perspective (with special reference to Delhi \& NCR)”, Indian Journal of Marketing, Vol. XXXVIII No. 6, pp. 42-9.

2. Bijmolt, T.H.A., Claassen, W. and Brus, B. (1998), "Children's understanding of TV advertising: effects of age, gender and parental influence”, Journal of Consumer Policy, Vol. 21, pp. 171-94.

3. Boush, D.M., Friestad, M. and Rose, G.M. (1994), “Adolescent skepticism towards TV advertising and knowledge of advertiser tactics", Journal of Consumer Research, Vol. 21 No. 1, pp. 165-75.

4. Byrd-Bredbenner, C. and Grasso, D. (1999), "Prime-time health: an analysis of health content in television commercials broadcast during programs viewed heavily by children", The International Electronic Journal of Health Education, Vol. 2 No. 4, pp. 159-69.

5. Consumers International (2004), "The junk food generation - a multi-country survey of the influence of television advertisements on children”, available at: www.epicpolicy.org/../thejunk-food-generation-a-multi-country-surveyinfluencetelevision-advertisements-chil.

6. Consumers International (2008), "The junk food trap, marketing unhealthy food to children in Asia Pacific”, available at: www.consumersinternational.org .

7. Cook, D.T. (2009), “Knowing the child consumer: historical and conceptual insights on qualitative children's consumer research", Young Consumers, Vol. 10 No. 4, pp. 269-282.

8. De La Ville, V.I., Brouge re, G. and Boireau, N. (2010), "How can food become fun? Exploring and testing possibilities", Young Consumers, Vol. 11No. 2, pp. 117-130

9. George, A. (2003), "Your 'TV baby', ad man's delight”, available at: www.hindu.com/thehindu/ mp/2003/12/22/stories/2003122201400200.htm.

10. Gillespie, A.H. (1989), “Comparison of family interaction patterns related to food and nutrition”, Journal of the American Dietetic Association, Vol. 89, pp. 509-12.

11. Haroon, M., Qureshi, T. M., Zia-ur-Rehman, M., \&Nisar, M. (2011). Does the food advertisement on television have the impact on children's food purchasing behavior? A study based on Pakistan food advertisement. International Journal of Business and Management, 6(1), 283.

12. Hardy, L.L., Baur, L.A., Garneett, S.P., Crawford, D., Campbell, K.J., Shrewsbury, V.A., Cowell, C.T. and Salmon, J. (2006), "Family and home correlates of television viewing in 12-13 year old adolescents: the Napean study", International Journal of Behavioural Nutrition and Physical Activity, Vol. 3 No. 24, available at: www.ijbnpa.org/content/3/1/24

13. Hingle, M.D., Castonguay, J.S., Ambuel, D.A., Smith, R.M. and Kunkel, D. (2015), “Alignment of children's food advertising with proposed federal guidelines", American Journal of Preventive Medicine, Vol. 48 No. 6, pp. 707-713 
14. Jenkin, G., Wilson, N. and Hermanson, N. (2008), “Identifying 'unhealthy' food advertising on television: a case study applying the UK nutrient profile model", Public Health Nutrition, Vol. 12 No. 5, pp. 614-23.

15. John, D.R. (1999), "Consumer socialization of children: a retrospective look at twenty-five years of research", Journal of Consumer Research, Vol. 26 No. 3, pp. 183-213.

16. Kaiser Family Foundation (2004), The Role of Media in Childhood Obesity, Kaiser Family Foundation, Menlo Park, CA, available at: www.kff.org/entmedia/upload/The-Role-Of-Media-in-Childhood-Obesity.pdf .

17. Kaur, Amandeep.(2011). Impact of Television advertising on children: A behavioural study.20-120

18. Kaur, P. (2011), "Foods promotion to children: understanding the need of responsibility in marketing to children", Information Management and Business Review, Vol. 2 No. 4, pp. 133-7.

19. Kelly, B., Halford, J.C.G., Boyland, E.J., Chapman, K., Bautista-Castañ o, I., Berg, C., Caroli, M., Cook, B., Coutinho, J.G., Effertz, T., Grammatikaki, E., Keller, K., Leung, R., Manios, Y., Monteiro, R., Pedley, C., Prell, H., Raine, K., Recine, E. and Serra-Majem, L. (2010), “Television food advertising to children: a global perspective”, American Journal of Public Health, Vol. 100 No. 9, pp. 1730-1736.

20. Kotz, K. and Story, M. (1994), “Food advertisements during children's saturday morning television programming: are they consistent with dietary recommendations?”, Journal of American Dietic Association, Vol. 94, pp. 1296-300.

21. Maher, J.K., Lord, J.B., Hughner, R.S. and Childs, N.M. (2006), “Food advertising on children's television”, Young Consumers, Vol. 7 No. 4, pp. 41-52.

22. Mcalister, A.R. and Bargh, D. (2016), "Dissuasion: the elaboration likelihood model and young children”, Young Consumers, Vol. 17 No. 3, pp. 210-225.

23. Mittal, M., Daga, A., Chhabara, G. and Lilani, J. (2010), "Parental perception of television advertisement on children's buying behaviour”, The IUP Journal of Marketing Management, Vol. IX No. 1, pp. 41-53.

24. Rozendaal, E., Opree, S.J. and Buijzen, M. (2016), “Development and validation of a survey instrument to measure children's advertising literacy", Media Psychology, Vol. 19 No. 1, pp. 72-100.

25. Sharma, A., \&Sonwaney, V. (2014). Theoretical modeling of influence of children on family purchase decision making. Procedia-Social and Behavioral Sciences, 133, 38-46.

26. Soni, P., \& Singh, R. (2012). Mediation of TV advertising to children. Society and Business Review.

27. Stitt, C. and Kunkel, D. (2008), "Food advertising during children's television programming on broadcast and cable channels", Health Communication, Vol. 23, pp. 573-84. 
